\title{
DIFFERENTIAL TRANSFORMS OF CESÀRO AVERAGES IN WEIGHTED SPACES
}

\author{
A. L. Bernardis and F. J. Martín-Reyes
}

Abstract

In this paper we obtain convergence results for the series of differences of Cesàro averages along lacunary sequences in the setting of weighted $L^{p}$-spaces. These results give some information about how the Cesàro averages converge. The paper extends results of an earlier work by R. L. Jones and J. Rosenblatt. The operators considered are essentially convolution operators given by kernels more singular than the ones in the article by Jones and Rosenblatt.

\section{Introduction}

The Lebesgue's Differentiation theorem in the real line establishes that if $f$ is locally integrable then

$$
\lim _{\varepsilon \rightarrow 0^{+}} \frac{1}{\varepsilon} \int_{x}^{x+\varepsilon} f(t) d t=f(x)
$$

for almost every $x$. We can interpret the above limit as Cesàro $(C, 1)$ right-continuity of $f$ at $x$ (see [7]). In general, for $\alpha>-1$, we say that $f$ is $(C, 1+\alpha)$ right-continuous at $x$ if

$$
\lim _{\varepsilon \rightarrow 0^{+}} D_{\varepsilon} f(x)=f(x),
$$

where the Cesàro- $\alpha$ averages $D_{\varepsilon} f$ are defined by

$$
D_{\varepsilon} f(x)=\frac{1+\alpha}{\varepsilon^{1+\alpha}} \int_{x}^{x+\varepsilon}(x+\varepsilon-t)^{\alpha} f(t) d t .
$$

2000 Mathematics Subject Classification. 42B25.

Key words. Differential transforms, Cesàro averages, weight.

This research has been partially supported by Spanish goverment Grant MTM20058350-C03-02. The first author was supported in part by CAI+D-UNL and CONICET. The second author was supported by Junta de Andalucía Grant FQM 354. 
If $\alpha=0$ then the Cesàro- $\alpha$ averages are the usual averages. It is easy to see that the $(C, 1+\alpha)$ right-continuity of $f$ at $x$ implies the $(C, 1+$ $\beta$ ) right-continuity of $f$ at $x$ for all $\beta>\alpha>-1$.

We are interested in obtaining information about the convergence of $D_{\varepsilon} f$. In this paper we are going to study the convergence through lacunary sequences.

Let us consider the Cesàro averages associated to a lacunary sequence, that is,

$$
D_{k} f(x)=D_{\varepsilon_{k}} f(x)=\frac{1+\alpha}{\varepsilon_{k}^{1+\alpha}} \int_{x}^{x+\varepsilon_{k}}\left(x+\varepsilon_{k}-t\right)^{\alpha} f(t) d t,
$$

where $\left\{\varepsilon_{k}\right\}_{k \in \mathbb{Z}}$ is a $\rho$-lacunary sequence of positive numbers, that is, $\varepsilon_{k+1} / \varepsilon_{k} \geq \rho>1$ for all $k \in \mathbf{Z}$. In order to study the limit of the averages $D_{k}$, it is natural to consider the maximal operator

$$
\mathcal{M}_{\alpha, k}^{+} f(x)=\sup _{k \in \mathbb{Z}} D_{k}|f|(x) .
$$

From known results about approximations of the identity we have that the operator $\mathcal{M}_{\alpha, k}^{+}$is of weak type $(1,1)$ and of strong type $(p, p)$ for $p>1$. As a consequence, we have that if $p>1$ and $f \in L^{p}(d x)$ then $\lim _{k \rightarrow-\infty} D_{k} f=f$ and $\lim _{k \rightarrow+\infty} D_{k} f=0$ almost everywhere and in the $L^{p}(d x)$-norm. For the limit case $p=1$ it is proved that $\lim _{k \rightarrow-\infty} D_{k} f=f$ and $\lim _{k \rightarrow+\infty} D_{k} f=0$ almost everywhere and in measure for all $f \in$ $L^{1}(d x)$. However, it follows from the results in $[\mathbf{7}]$ that the maximal operator

$$
\mathcal{M}_{\alpha}^{+} f(x)=\sup _{\varepsilon>0} D_{\varepsilon}|f|(x)
$$

is of strong type $(p, p)$ for $p>1 /(1+\alpha)$, it is of restricted weak type $\left(\frac{1}{1+\alpha}, \frac{1}{1+\alpha}\right)$ and it is not of weak type $\left(\frac{1}{1+\alpha}, \frac{1}{1+\alpha}\right)$ for $\alpha<0$. (Notice that if $\alpha \geq 0$, the operator $\mathcal{M}_{\alpha}^{+}$is pointwise equivalent to the onesided Hardy-Littlewood maximal operator $\mathcal{M}_{0}^{+}$which we simply denote by $M^{+}$. For that reason we are considering only the case $\alpha \leq 0$.) The study of the boundedness of $\mathcal{M}_{\alpha}^{+}$on weighted spaces can be found in [11] (see also [2]). In the setting of Ergodic Theory the convergence of Cesàro- $\alpha$ averages appears, for instance, in $[\mathbf{3}]$ and $[\mathbf{5}]$.

As we said above, we wish to obtain some information about how the convergence of $D_{k} f$ occurs. In [6] Jones and Rosenblatt discuss the problem for the usual averages (case $\alpha=0$ ) in connection with the convergence of ergodic averages. To obtain information about how the convergence of the sequence $\left\{D_{k}\right\}$ occurs they study the behavior of the 
series of the differences

$$
\sum_{k=-\infty}^{\infty} v_{k}\left[D_{k} f(x)-D_{k-1} f(x)\right]
$$

where $\left\{v_{k}\right\}$ is a bounded sequence of real or complex numbers (we shall say that $\left\{v_{k}\right\}$ is a multiplying sequence and we shall write $\left\|v_{k}\right\|_{\infty}=$ $\left.\sup _{k}\left|v_{k}\right|\right)$. In [6], Jones and Rosenblatt work in Lebesgue spaces $L^{p}(d x)$ while in $[\mathbf{1}]$ the results were extended to weighted Lebesgue spaces.

In order to make precise statements about the convergence properties of (1.2), we begin by pointing out that $D_{k} f(x)=\varphi_{k} * f(x)$ where $\varphi_{k}(x)=$ $\frac{1}{\varepsilon_{k}} \varphi\left(x / \varepsilon_{k}\right)$ and $\varphi(x)=(1+\alpha)(1+x)^{\alpha} \chi_{(-1,0)}(x)$. For each $N \in \mathbb{Z}^{2}$, $N=\left(N_{1}, N_{2}\right)$ with $N_{1}<N_{2}$ we define the sum

$$
T_{N}^{\alpha} f(x)=\sum_{k=N_{1}}^{N_{2}} v_{k}\left[D_{k} f(x)-D_{k-1} f(x)\right]=K_{N}^{\alpha} * f(x),
$$

where

$$
K_{N}^{\alpha}(x)=\sum_{k=N_{1}}^{N_{2}} v_{k}\left[\varphi_{k}(x)-\varphi_{k-1}(x)\right]
$$

Our goal is to prove convergence results of $T_{N}^{\alpha} f(x)$ as $N=\left(N_{1}, N_{2}\right)$ tends to $(-\infty,+\infty)$ which means that $N_{1} \rightarrow-\infty$ and $N_{2} \rightarrow+\infty$. As usual, to prove the a.e. convergence, we shall study the boundedness of the associated maximal operator

$$
T^{*} f(x)=\sup _{N \in \mathbb{Z}^{2}}\left|T_{N}^{\alpha} f(x)\right|
$$

in the setting of the weighted spaces $L^{p}(w)$, that is, the space of all measurable functions $f$ such that $\|f\|_{L^{p}(w)}=\left(\int_{\mathbb{R}}|f|^{p} w\right)^{1 / p}<\infty$.

Since the operators $T_{N}^{\alpha}$ are convolution operators with kernels $K_{N}^{\alpha}$ supported in $(-\infty, 0)$, the weights that appear are the one sided $A_{p}^{+}$weights defined by E. Sawyer $[\mathbf{1 2}]$. We say that a weight $w$ belongs to the class $A_{1}^{+}[\mathbf{1 2}],[\mathbf{9}]$, if there exists $C$ such that

$$
M^{-} w(x) \leq C w(x) \quad \text { a.e., }
$$

where $M^{-}$is the left-sided Hardy-Littlewood maximal function defined as

$$
M^{-} f(x)=\sup _{\varepsilon>0} \frac{1}{\varepsilon} \int_{0}^{\varepsilon}|f(x-t)| d t
$$


For $1<p<\infty$, we say that $w$ belongs to $A_{p}^{+}[\mathbf{1 2}]$ if there exists $C$ such that for any three points $a<b<c$

$$
\left(\int_{a}^{b} w\right)^{\frac{1}{p}}\left(\int_{b}^{c} w^{1-p^{\prime}}\right)^{\frac{1}{p}} \leq C(c-a),
$$

where $p+p^{\prime}=p p^{\prime}$.

The next theorem contains a relation between the maximal operator $\mathcal{M}_{\alpha}^{+}$and $A_{p}^{+}$classes (see [11] and [2]). We shall use this theorem in our proofs.

Theorem 1.4. Let $-1<\alpha \leq 0$. If $1 /(1+\alpha)<p<\infty$ and $w \in A_{p(1+\alpha)}^{+}$ then there exists a constant $C$ such that

$$
\int\left|\mathcal{M}_{\alpha}^{+} f(x)\right|^{p} w(x) d x \leq C \int|f(x)|^{p} w(x) d x
$$

for all $f \in L^{p}(w)$.

In what follows, we state the main results of this paper.

Theorem 1.6. Let $\left\{\varepsilon_{k}\right\}$ be a $\rho$-lacunary sequence, $\left\{v_{k}\right\}$ a multiplying sequence and $-1<\alpha \leq 0$. If $1 /(1+\alpha)<p<\infty$ and $w \in A_{p(1+\alpha)}^{+}$then there exists a constant $C$ such that

$$
\left\|T^{*} f\right\|_{L^{p}(w)} \leq C\|f\|_{L^{p}(w)},
$$

for all functions $f \in L^{p}(w)$.

Theorem 1.7. Let $\left\{\varepsilon_{k}\right\}$ be a $\rho$-lacunary sequence, $\left\{v_{k}\right\}$ a multiplying sequence and $-1<\alpha \leq 0$. If $w^{r} \in A_{1}^{+}$for some $r>1 /(1+\alpha)$ then there exists a constant $C$ such that

$$
w\left(\left\{x \in \mathbb{R}:\left|T^{*} f(x)\right|>\lambda\right\}\right) \leq \frac{C}{\lambda}\|f\|_{L^{1}(w)},
$$

for all $\lambda>0$ and all functions $f \in L^{1}(w)$.

Notice that, under the assumptions of the last theorem, that is, $w^{r} \in$ $A_{1}^{+}$for some $r>1 /(1+\alpha)$, we have that $T^{*}$ is of strong type $(p, p)$ with respect to $w(x) d x$ for all $p>1$. This statement is easily seen applying the last two theorems and interpolation arguments.

Remark 1.8. It follows from Theorem 1.4 and the arguments in the proof of Theorem 1.7 that the conclusions of Theorems 1.6 and 1.7 hold for the maximal operator $\mathcal{M}_{\alpha, k}^{+}$.

Using the theorems and proving the a.e. convergence in the Schwartz class we obtain the following result. 
Theorem 1.9. Let $\left\{\varepsilon_{k}\right\}$ be a $\rho$-lacunary sequence and let $\left\{v_{k}\right\}$ be a multiplying sequence. Assume that $w$ is a weight.

(i) If $-1<\alpha \leq 0,1 /(1+\alpha)<p<\infty$ and $w \in A_{p(1+\alpha)}^{+}$then $T_{N}^{\alpha} f$ converge a.e. and in $L^{p}(w)$-norm for all $f \in L^{p}(w)$ as $N=$ $\left(N_{1}, N_{2}\right)$ tends to $(-\infty,+\infty)$.

(ii) If $w^{r} \in A_{1}^{+}$for some $r>1 /(1+\alpha)$ then $T_{N}^{\alpha} f$ converge a.e. and in measure for all $f \in L^{1}(w)$ as $N=\left(N_{1}, N_{2}\right)$ tends to $(-\infty,+\infty)$.

We remark that applying Theorems 1.6 and 1.7 with $w \equiv 1$ we have that $T^{*}$ is of weak type $(1,1)$ and of strong type $(p, p), 1<p<\infty$, with respect to the Lebesgue measure. The corresponding results of convergence also holds.

The organization of the paper is as follows. Section 2 is devoted to state properties of the lacunary sequences. In Section 3 we prove some properties of the kernels $K_{N}^{\alpha}$. Section 4 is devoted to prove uniform boundedness of the operators $T_{N}^{\alpha}$, while Theorems 1.6, 1.7, and 1.9 are proved in Sections 5, 6 and 7, respectively.

Throughout the paper, we shall use the notations introduced in this section and the letter $C$ will mean a positive constant not necessarily the same at each occurrence.

\section{Lacunary sequences}

We establish in this section some properties of the $\rho$-lacunary sequence $\left\{\varepsilon_{k}\right\}$. The next proposition shows that, without loss of generality, we may assume that

$$
1<\rho \leq \frac{\varepsilon_{k+1}}{\varepsilon_{k}} \leq \rho^{2} .
$$

Proposition 2.2. Given the $\rho$-lacunary sequence $\left\{\varepsilon_{k}\right\}$ and the multiplying sequence $\left\{v_{k}\right\}$, we can define a $\rho$-lacunary sequence $\left\{\eta_{k}\right\}$ and a multiplying sequence $\left\{w_{k}\right\}$ verifying the following properties:

(i) $1<\rho \leq \frac{\eta_{k+1}}{\eta_{k}} \leq \rho^{2}$ and $\left\|v_{k}\right\|_{\infty}=\left\|w_{k}\right\|_{\infty}$.

(ii) For all $N=\left(N_{1}, N_{2}\right)$ there exists $M=\left(M_{1}, M_{2}\right)$ with $T_{N}^{\alpha}=$ $\tilde{T}_{M}$, where $\tilde{T}_{M}$ is the operator defined in (1.3) for the new sequences $\left\{\eta_{k}\right\}$ and $\left\{w_{k}\right\}$.

The proof is exactly as in the case $\alpha=0$ (see [1] ).

It follows from this proposition that it is enough to prove all the results of this paper in the case of a $\rho$-lacunary sequence satisfying (2.1). For 
this reason, in the rest of the paper we assume that $\left\{\varepsilon_{k}\right\}$ satisfies $(2.1)$ without saying it explicitly. Observe that, under this assumption, the following properties hold:

$$
\left(\frac{1}{\rho}\right)^{2(m-n)} \leq \frac{\varepsilon_{n}}{\varepsilon_{m}} \leq\left(\frac{1}{\rho}\right)^{m-n}, \quad \text { for all } m>n .
$$

In fact, these inequalities follow from (2.1) and the equality

$$
\frac{\varepsilon_{n}}{\varepsilon_{m}}=\frac{\varepsilon_{n}}{\varepsilon_{n+1}} \frac{\varepsilon_{n+1}}{\varepsilon_{n+2}} \ldots \frac{\varepsilon_{m-1}}{\varepsilon_{m}} .
$$

If we denote by $\beta$ the smallest positive integer such that

$$
1 / \rho+(1 / \rho)^{\beta} \leq 1,
$$

we get from (2.3) that

$$
\varepsilon_{i}+\varepsilon_{m} \leq \varepsilon_{m+1} \quad \text { for all } m \geq i+\beta-1 .
$$

\section{Properties of the kernels $K_{N}^{\alpha}$}

The next lemma will allow us to prove the uniform boundedness on $L^{2}(d x)$ of the operators $T_{N}^{\alpha}$.

Lemma 3.1. Let $-1<\alpha \leq 0$ and $\varphi(x)=(1+\alpha)(1+x)^{\alpha} \chi_{(-1,0)}(x)$. The Fourier transform of $\varphi$, defined by

$$
\hat{\varphi}(\xi)=\int_{-\infty}^{\infty} \varphi(x) \exp (-i x \xi) d x,
$$

has the following properties:

(i) There exists a constant $C$ depending on $\alpha$ such that $|\hat{\varphi}(\xi)| \leq \frac{C}{\mid \xi^{1+\alpha}}$ for all $\xi$ such that $|\xi|>1$.

(ii) $\left|\frac{d \hat{\varphi}}{d \xi}(\xi)\right| \leq 1$ for all $\xi$.

Proof: Since (ii) is obvious, we shall only prove (i). Let $\xi$ be such that $|\xi|>1$. Then

$$
\begin{aligned}
\frac{|\hat{\varphi}(\xi)|}{1+\alpha} & \leq\left|\int_{-1}^{-1+\frac{1}{|\xi|}}(1+x)^{\alpha} \exp (-i x \xi) d x\right|+\left|\int_{-1+\frac{1}{|\xi|}}^{0}(1+x)^{\alpha} \exp (-i x \xi) d x\right| \\
& =I+I I .
\end{aligned}
$$

Clearly

$$
I \leq \int_{-1}^{-1+\frac{1}{|\xi|}}(1+x)^{\alpha} d x=\frac{1}{(1+\alpha)|\xi|^{1+\alpha}}
$$


Integrating by parts

$$
\begin{aligned}
I I \leq \mid & -\frac{1}{i \xi}+\frac{1}{i \xi|\xi|^{\alpha}} \exp \left(-i \xi\left(-1+\frac{1}{|\xi|}\right)\right) \\
& +\frac{\alpha}{i \xi} \int_{-1+\frac{1}{|\xi|}}^{0}(1+x)^{\alpha-1} \exp (-i x \xi) d x \mid \\
\leq & \frac{1}{|\xi|}+\frac{1}{|\xi|^{\alpha+1}}+\frac{1}{|\xi|}\left(1+\frac{1}{|\xi|^{\alpha}}\right) \leq \frac{4}{|\xi|^{\alpha+1}} .
\end{aligned}
$$

Lemma 3.2. Let $-1<\alpha \leq 0$. There exists a constant $C$ depending only on $\alpha, \rho$ and $\left\|v_{k}\right\|_{\infty}$ such that

$$
\sup _{N}\left|\widehat{K_{N}^{\alpha}}(\xi)\right|=\sup _{N}\left|\sum_{k=N_{1}}^{N_{2}} v_{k}\left(\hat{\varphi}\left(\varepsilon_{k} \xi\right)-\hat{\varphi}\left(\varepsilon_{k-1} \xi\right)\right)\right| \leq C
$$

for all $\xi \in \mathbb{R}$.

Proof: Clearly $\left|\widehat{K_{N}^{\alpha}}(0)\right|=0$. For fixed $\xi \in \mathbb{R}, \xi \neq 0$, let $k_{0}$ be such that $\varepsilon_{k_{0}-1}<1 /|\xi| \leq \varepsilon_{k_{0}}$. Then

$$
\begin{aligned}
\left|\widehat{K_{N}^{\alpha}}(\xi)\right| & \leq \sum_{k=-\infty}^{\infty}\left|v_{k}\right|\left|\hat{\varphi}\left(\varepsilon_{k} \xi\right)-\hat{\varphi}\left(\varepsilon_{k-1} \xi\right)\right| \\
& \leq\left\|v_{k}\right\|_{\infty} \sum_{k=-\infty}^{k_{0}} \cdots+\left\|v_{k}\right\|_{\infty} \sum_{k=k_{0}+1}^{\infty} \cdots=I+I I .
\end{aligned}
$$

To estimate $I$ notice that, by using the mean value theorem, the second property in Lemma 3.1 and (2.1), we get that

$$
\left|\hat{\varphi}\left(\varepsilon_{k} \xi\right)-\hat{\varphi}\left(\varepsilon_{k-1} \xi\right)\right| \leq C\left(\varepsilon_{k}-\varepsilon_{k-1}\right)|\xi| \leq C\left(\rho^{2}-1\right) \varepsilon_{k-1}|\xi| .
$$

Then, using (2.3) and by the election of $k_{0}$ we get

$I \leq C\left\|v_{k}\right\|_{\infty}|\xi|\left(\rho^{2}-1\right) \sum_{k=-\infty}^{k_{0}} \varepsilon_{k-1} \leq C\left\|v_{k}\right\|_{\infty}|\xi|\left(\rho^{2}-1\right) \varepsilon_{k_{0}-1} \sum_{i=0}^{+\infty}\left(\frac{1}{\rho}\right)^{i} \leq C$. 
To estimate $I I$ we observe that if $k \geq k_{0}+1$ then $\left|\xi \varepsilon_{k}\right| \geq\left|\xi \varepsilon_{k-1}\right| \geq$ $\left|\xi \varepsilon_{k_{0}}\right| \geq 1$. Therefore, by Lemma 3.1 and the election of $k_{0}$,

$$
\begin{aligned}
I I & \leq\left\|v_{k}\right\|_{\infty} \sum_{k=k_{0}+1}^{\infty}\left(\left|\hat{\varphi}\left(\varepsilon_{k} \xi\right)\right|+\left|\hat{\varphi}\left(\varepsilon_{k-1} \xi\right)\right|\right) \\
& \leq \frac{C\left\|v_{k}\right\|_{\infty}}{|\xi|^{1+\alpha}} \sum_{k=k_{0}+1}^{\infty} \frac{1}{\varepsilon_{k-1}^{1+\alpha}} \\
& \leq \frac{C\left\|v_{k}\right\|_{\infty}}{|\xi|^{1+\alpha} \varepsilon_{k_{0}}^{1+\alpha}} \sum_{i=0}^{+\infty}\left(\frac{1}{\rho^{1+\alpha}}\right)^{i} \leq C .
\end{aligned}
$$

Lemma 3.3. Let $-1<\alpha \leq 0$. There exists a constant $C$ depending only on $\alpha, \rho$ and $\left\|v_{k}\right\|_{\infty}$ such that

$$
\begin{aligned}
\sup _{N}\left|K_{N}^{\alpha}(x)\right| & \leq \sum_{k=-\infty}^{+\infty}\left|v_{k}\left(\varphi_{k}(x)-\varphi_{k-1}(x)\right)\right| \\
& \leq \frac{C}{|x|}+C \sum_{k=-\infty}^{+\infty} \frac{\left(\varepsilon_{k+1}+x\right)^{\alpha}}{\varepsilon_{k+1}^{1+\alpha}} \chi_{\left(-\varepsilon_{k+1},-\varepsilon_{k}\right)}(x),
\end{aligned}
$$

for all $x \neq 0$. Consequently

$$
K^{\alpha}(x)=\sum_{k=-\infty}^{+\infty} v_{k}\left(\varphi_{k}(x)-\varphi_{k-1}(x)\right)
$$

is defined for all $x \in \mathbb{R}$ and

$$
\int_{\{x:|x|<R\}}|x|\left|K^{\alpha}(x)\right| d x \leq C R
$$

for all $R>0$; the same inequality holds for $K_{N}^{\alpha}$ with a constant independent of $N$.

Proof: It is clear that $K_{N}^{\alpha}(x)=0$ for $x \geq 0$. For negative $x$, there exists $k_{0}$ such that $-\varepsilon_{k_{0}+1} \leq x<-\varepsilon_{k_{0}}$, and since the sequence $\varepsilon_{k}$ is increasing, we get that $\chi_{\left(-\varepsilon_{k}, 0\right)}(x)=0$ for every $k \leq k_{0}$, and $\chi_{\left(-\varepsilon_{k}, 0\right)}(x)=1$ for 
every $k>k_{0}$. Thus we obtain

$$
\begin{aligned}
\left|K_{N}^{\alpha}(x)\right| & \leq \sum_{k=-\infty}^{+\infty}\left|v_{k}\left(\varphi_{k}(x)-\varphi_{k-1}(x)\right)\right| \\
& \leq C \sum_{k=k_{0}+1}^{\infty} \frac{\left(\varepsilon_{k}+x\right)^{\alpha}}{\varepsilon_{k}^{1+\alpha}} \\
& \leq C\left(\sum_{k=k_{0}+2}^{\infty} \frac{\left(\varepsilon_{k}-\varepsilon_{k_{0}+1}\right)^{\alpha}}{\varepsilon_{k}^{1+\alpha}}+\frac{\left(\varepsilon_{k_{0}+1}+x\right)^{\alpha}}{\varepsilon_{k_{0}+1}^{1+\alpha}}\right) \\
& \leq C\left(\sum_{k=k_{0}+2}^{\infty} \frac{1}{\varepsilon_{k}}+\frac{\left(\varepsilon_{k_{0}+1}+x\right)^{\alpha}}{\varepsilon_{k_{0}+1}^{1+\alpha}}\right) \\
& \leq \frac{C}{\varepsilon_{k_{0}+1}}+C \frac{\left(\varepsilon_{k_{0}+1}+x\right)^{\alpha}}{\varepsilon_{k_{0}+1}^{1+\alpha}} \\
& \leq \frac{C}{|x|}+C \frac{\left(\varepsilon_{k_{0}+1}+x\right)^{\alpha}}{\varepsilon_{k_{0}+1}^{1+\alpha}}
\end{aligned}
$$

To prove the integral inequality we take $k_{0}$ such that $\varepsilon_{k_{0}} \leq R \leq \varepsilon_{k_{0}+1}$. Then using the estimate (3.4) we get

$$
\begin{aligned}
\int_{\{x:|x|<R\}}|x|\left|K^{\alpha}(x)\right| d x & \leq C R+C \sum_{k=-\infty}^{k_{0}} \int_{-\varepsilon_{k+1}}^{-\varepsilon_{k}}|x| \frac{\left(\varepsilon_{k+1}+x\right)^{\alpha}}{\varepsilon_{k+1}^{1+\alpha}} d x \\
& \leq C R+C \sum_{k=-\infty}^{k_{0}} \int_{-\varepsilon_{k+1}}^{-\varepsilon_{k}} \frac{\left(\varepsilon_{k+1}+x\right)^{\alpha}}{\varepsilon_{k+1}^{\alpha}} d x \\
& \leq C R+C \sum_{k=-\infty}^{k_{0}} \varepsilon_{k} \leq C R+C \varepsilon_{k_{0}} \leq C R
\end{aligned}
$$

In what follows we shall prove that the kernels $K_{N}^{\alpha}$ verify a one-sided smoothness condition uniformly. The proof is similar to the one in $[\mathbf{1 3}]$. To prove this result we shall need the following lemma which follows easily taking into account the supports of the characteristic functions and using the property (2.4) of the sequence $\left\{\varepsilon_{k}\right\}$. We omit the proof. 
Lemma 3.5. Let $-1<\alpha \leq 0$. If $j \geq i+\beta, 0<x \leq \varepsilon_{i}, \varepsilon_{j}<y<\varepsilon_{j+1}$ and we write $\mathcal{F}_{k}(x, y)=\left(\varepsilon_{k}+x-y\right)^{\alpha} \chi_{\left(x, x+\varepsilon_{k}\right)}(y)-\left(\varepsilon_{k}-y\right)^{\alpha} \chi_{\left(0, \varepsilon_{k}\right)}(y)$. Then

$$
\mathcal{F}_{k}(x, y)= \begin{cases}0 & \text { if } k \leq j-1 \\ \left(\varepsilon_{j}+x-y\right)^{\alpha} \chi_{\left(\varepsilon_{j}, x+\varepsilon_{j}\right)}(y) & \text { if } k=j \\ \left(\varepsilon_{k}+x-y\right)^{\alpha}-\left(\varepsilon_{k}-y\right)^{\alpha} & \text { if } k>j .\end{cases}
$$

Now we state and prove the one-sided smoothness condition.

Lemma 3.6. Let $-1<\alpha \leq 0$. Let $1 \leq r<-1 / \alpha, j \geq i+\beta$ and $0<$ $x \leq \varepsilon_{i}$. Then there exists a constant $C$ depend only on $\alpha, r$ and $\left\|v_{k}\right\|_{\infty}$ such that

$$
\left(\int_{\varepsilon_{j}}^{\varepsilon_{j+1}}\left|K_{N}^{\alpha}(x-y)-K_{N}^{\alpha}(-y)\right|^{r} d y\right)^{1 / r} \leq C \frac{\varepsilon_{j}^{1 / r-1}}{\rho^{(j-i)(\alpha+1 / r)}} .
$$

Proof: We may assume $\alpha<0$. The case $\alpha=0$ can be found in [1]. First, notice that applying the previous lemma

$$
\begin{aligned}
\frac{\left|K_{N}^{\alpha}(x-y)-K_{N}^{\alpha}(-y)\right|}{1+\alpha} \leq & 2\left\|v_{k}\right\|_{\infty} \sum_{k=N_{1}-1}^{N_{2}} \frac{1}{\varepsilon_{k}^{1+\alpha}}\left|\mathcal{F}_{k}(x, y)\right| \\
\leq & C \frac{1}{\varepsilon_{j}^{1+\alpha}}\left(\varepsilon_{j}+x-y\right)^{\alpha} \chi_{\left(\varepsilon_{j}, x+\varepsilon_{j}\right)}(y) \\
& +C \sum_{k>j} \frac{1}{\varepsilon_{k}^{1+\alpha}}\left|\left(\varepsilon_{k}+x-y\right)^{\alpha}-\left(\varepsilon_{k}-y\right)^{\alpha}\right| .
\end{aligned}
$$

Then we get that the left hand side in (3.7) is bounded by the sum of the following two terms:

$$
I=C \frac{1}{\varepsilon_{j}^{1+\alpha}}\left(\int_{\varepsilon_{j}}^{x+\varepsilon_{j}}\left(\varepsilon_{j}+x-y\right)^{r \alpha} d y\right)^{1 / r}
$$

and

$$
I I=C \sum_{k=j+1}^{\infty} \frac{1}{\varepsilon_{k}^{1+\alpha}}\left(\int_{\varepsilon_{j}}^{\varepsilon_{j+1}}\left|\left(\varepsilon_{k}+x-y\right)^{\alpha}-\left(\varepsilon_{k}-y\right)^{\alpha}\right|^{r} d y\right)^{1 / r} .
$$

Since $r<-1 / \alpha$, computing the integral in $I$ and using (2.3) we obtain that

$$
I \leq C \frac{1}{\varepsilon_{j}^{1+\alpha}} x^{\alpha+1 / r} \leq C \frac{\varepsilon_{i}^{\alpha+1 / r}}{\varepsilon_{j}^{1+\alpha}} \leq \frac{C \varepsilon_{j}^{1 / r-1}}{\rho^{(j-i)(\alpha+1 / r)}} .
$$


Now we estimate $I I$. Observe that

$I I \leq C \frac{1}{\varepsilon_{j+1}^{1+\alpha}}\left(\int_{\varepsilon_{j}}^{\varepsilon_{j+1}-\varepsilon_{i}} \cdots\right)^{1 / r}+C \frac{1}{\varepsilon_{j+1}^{1+\alpha}}\left(\int_{\varepsilon_{j+1}-\varepsilon_{i}}^{\varepsilon_{j+1}} \ldots\right)^{1 / r}+C \sum_{k=j+2}^{\infty} \cdots$

Then, applying the mean value theorem in the first term and in each term of the sum in the above inequality we get

$$
\begin{aligned}
I I \leq & C \frac{|x|}{\varepsilon_{j+1}^{1+\alpha}}\left(\int_{\varepsilon_{j}}^{\varepsilon_{j+1}-\varepsilon_{i}}\left(\varepsilon_{j+1}-y\right)^{(\alpha-1) r} d y\right)^{1 / r} \\
& +C \frac{1}{\varepsilon_{j+1}^{1+\alpha}}\left(\int_{\varepsilon_{j+1}-\varepsilon_{i}}^{\varepsilon_{j+1}}\left|\left(\varepsilon_{j+1}+x-y\right)^{\alpha}-\left(\varepsilon_{j+1}-y\right)^{\alpha}\right|^{r} d y\right)^{1 / r} \\
& +C \sum_{k=j+2}^{\infty} \frac{|x|}{\varepsilon_{k}^{1+\alpha}}\left(\int_{\varepsilon_{j}}^{\varepsilon_{j+1}}\left(\varepsilon_{k}-y\right)^{(\alpha-1) r} d y\right)^{1 / r}=I I I+I V+V
\end{aligned}
$$

To estimate $I I I$, we compute the integral taking into account that ( $\alpha-$ 1) $r+1<0$ so that

$$
\begin{aligned}
I I I & \leq C \frac{|x|}{\varepsilon_{j+1}^{1+\alpha}}\left(\frac{1}{(\alpha-1) r+1}\left[\left(\varepsilon_{j+1}-\varepsilon_{j}\right)^{(\alpha-1) r+1}-\varepsilon_{i}^{(\alpha-1) r+1}\right]\right)^{1 / r} \\
& \leq C \frac{|x|}{\varepsilon_{j+1}^{1+\alpha}} \varepsilon_{i}^{(\alpha-1)+1 / r} \leq C \frac{\varepsilon_{i}^{\alpha+1 / r}}{\varepsilon_{j+1}^{1+\alpha}} \leq \frac{C \varepsilon_{j}^{1 / r-1}}{\rho^{(j-i)(\alpha+1 / r)}}
\end{aligned}
$$

On the other hand,

$$
I V \leq \frac{C}{\varepsilon_{j+1}^{1+\alpha}}\left(\int_{\varepsilon_{j+1}-\varepsilon_{i}}^{\varepsilon_{j+1}}\left(\varepsilon_{j+1}-y\right)^{\alpha r} d y\right)^{1 / r} \leq \frac{C \varepsilon_{i}^{\alpha+1 / r}}{\varepsilon_{j+1}^{1+\alpha}} \leq \frac{C \varepsilon_{j}^{1 / r-1}}{\rho^{(j-i)(\alpha+1 / r)}}
$$

Finally

$$
V \leq C \sum_{k=j+2}^{\infty} \frac{|x|}{|(\alpha-1) r+1| \varepsilon_{k}^{1+\alpha}}\left(\varepsilon_{k}-\varepsilon_{j+1}\right)^{(\alpha-1)+1 / r}
$$


Since $k \geq j+2$ we have that $\varepsilon_{k}-\varepsilon_{j+1} \geq C \varepsilon_{j}$. Therefore

$$
\begin{aligned}
V & \leq C \sum_{k=j+2}^{\infty} \frac{|x|}{\varepsilon_{k}^{1+\alpha}} \varepsilon_{j}^{(\alpha-1)+1 / r}=C|x| \varepsilon_{j}^{1 / r-2} \\
& \leq C \frac{\varepsilon_{i}}{\varepsilon_{j}} \varepsilon_{j}^{1 / r-1} \leq C\left(\frac{1}{\rho^{j-i}}\right)^{\alpha+1 / r} \varepsilon_{j}^{1 / r-1} \leq \frac{C \varepsilon_{j}^{1 / r-1}}{\rho^{(j-i)(\alpha+1 / r)}} .
\end{aligned}
$$

Remark 3.8. It follows from the proof of the above lemma that if $N=$ $\left(N_{1}, N_{2}\right)$ with $N_{2}<i+\beta \leq j$ then

$$
\left|K_{N}^{\alpha}(x-y)-K_{N}^{\alpha}(-y)\right|=0
$$

for all $x \in\left(0, \varepsilon_{i}\right]$ and $y \in\left(\varepsilon_{j}, \varepsilon_{j+1}\right)$.

Remark 3.9. Lemma 3.6 does not hold for $r=-1 / \alpha$. In order to prove this statement, let us take $v_{k}=1$ for all $k$. Let us fix $i \in \mathbb{Z}, j \geq i+\beta$ and $x \in\left(0, \varepsilon_{i}\right)$. We choose $N=\left(N_{1}, N_{2}\right)$ such that $x+\varepsilon_{N_{1}-1}<\varepsilon_{i}$ and $N_{2} \geq i+\beta$. Then for all $y \in\left(\varepsilon_{j}, \varepsilon_{j+1}\right)$

$$
\begin{aligned}
K_{N}^{\alpha}(x-y)-K_{N}^{\alpha}(-y)= & (1+\alpha) \frac{\left(\varepsilon_{N_{2}}+x-y\right)^{\alpha}}{\varepsilon_{N_{2}}^{1+\alpha}} \chi_{\left(x, x+\varepsilon_{N_{2}}\right)}(y) \\
& -(1+\alpha) \frac{\left(\varepsilon_{N_{2}}-y\right)^{\alpha}}{\varepsilon_{N_{2}}^{1+\alpha}} \chi_{\left(0, \varepsilon_{N_{2}}\right)}(y) .
\end{aligned}
$$

Therefore for $j=N_{2}$,

$$
\begin{aligned}
\int_{\varepsilon_{j}}^{\varepsilon_{j+1}}\left|K_{N}^{\alpha}(x-y)-K_{N}^{\alpha}(-y)\right|^{-1 / \alpha} d y \\
=(1+\alpha) \int_{\varepsilon_{N_{2}}}^{x+\varepsilon_{N_{2}}}\left(\frac{\left(\varepsilon_{N_{2}}+x-y\right)^{\alpha}}{\varepsilon_{N_{2}}^{1+\alpha}}\right)^{-1 / \alpha} d y=+\infty .
\end{aligned}
$$

The condition in Lemma 3.6 is called the one-sided $D_{r}$ condition. With this notation, we have proved that for every $r \in[1,-1 / \alpha)$ the kernels $K_{N}^{\alpha}$ satisfy the one-sided condition $D_{r}$ uniformly on $N$ and they do not satisfy $D_{-1 / \alpha}$ in general. Condition $D_{1}$ is essentially Hörmander's condition as we prove in the next corollary. 
Corollary 3.10. Let $-1<\alpha \leq 0$. The kernels $K_{N}^{\alpha}$ satisfy the following Hörmander's condition uniformly on $N$ : there exists a constant $C$ depending only on $\alpha, \rho$ and $\left\|v_{k}\right\|_{\infty}$ such that

$$
\int_{|y|>C_{\rho}|x|}\left|K_{N}^{\alpha}(x-y)-K_{N}^{\alpha}(-y)\right| d y \leq C, \quad x \in \mathbb{R},
$$

where $C_{\rho}=\rho^{2(\beta+1)}$ and $x \neq 0$.

Proof: Given $x \in \mathbb{R} \backslash\{0\}$ let $i \in \mathbb{Z}$ such that $\varepsilon_{i-1}<|x| \leq \varepsilon_{i}$. It is clear by the lacunarity of the sequence $\left\{\varepsilon_{j}\right\}$ that

$$
\left\{y:|y|>C_{\rho}|x|\right\} \subset\left\{y:|y|>C_{\rho} \varepsilon_{i-1}\right\} \subset\left\{y:|y|>\varepsilon_{i+\beta}\right\} .
$$

Now, let us observe that if $\varepsilon_{i-1}<|x| \leq \varepsilon_{i}$ and $-\varepsilon_{j+1}<y \leq-\varepsilon_{j}$ with $j \geq i+\beta$, then $K_{N}^{\alpha}(x-y)=K_{N}^{\alpha}(-\bar{y})=0$. So that we only need to consider $y>0$. Thus

$\int_{|y|>C_{\rho}|x|}\left|K_{N}^{\alpha}(x-y)-K_{N}^{\alpha}(-y)\right| d y \leq \int_{y>\varepsilon_{i+\beta}}\left|K_{N}^{\alpha}(x-y)-K_{N}^{\alpha}(-y)\right| d y$.

Now, if $x>0$, the lemma follows simply from Lemma 3.3 with $r=1$. If $x<0$, by a change of variables we can obtain the same boundedness.

\section{Boundedness of the operators $T_{N}^{\alpha}$}

This section is devoted to prove the uniform boundedness of the operators $T_{N}^{\alpha}$. First, notice that since the kernels $K_{N}^{\alpha}$ satisfy the Hörmander's condition uniformly and their Fourier transforms are uniformly bounded in $L^{\infty}$, we have the following result (see, for example, Lemma II.6.1 in $[4])$.

Theorem 4.1. The operators $T_{N}^{\alpha}$ verify the following inequalities with constants independent of $N$ and $f$.

(i) For all $\lambda>0,\left|\left\{x \in \mathbb{R}:\left|T_{N}^{\alpha} f(x)\right|>\lambda\right\}\right| \leq \frac{C}{\lambda}\|f\|_{L^{1}}$.

(ii) For $1<p<\infty,\left\|T_{N}^{\alpha} f\right\|_{L^{p}} \leq C_{p}\|f\|_{L^{p}}$.

The study of the operators $T_{N}^{\alpha}$ in weighted spaces requires the introduction of the following one-sided maximal functions:

$$
M_{s}^{+} f(x)=\sup _{h>0}\left(\frac{1}{h} \int_{x}^{x+h}|f(y)|^{s} d y\right)^{1 / s} \quad(1 \leq s<\infty)
$$

and

$$
f^{+, \#}(x)=\sup _{h>0} \frac{1}{h} \int_{x}^{x+h}\left(f(y)-\frac{1}{h} \int_{x+h}^{x+2 h} f\right)^{+} d y
$$


where $f$ is a locally integrable function taking values in $\mathbb{R}$ and $z^{+}$stands for the positive part of the real number $z$. We shall also need the following theorem that was proved in $[\mathbf{1 0}]$.

Theorem 4.2. For any $0<p<\infty$ and $w \in A_{\infty}^{+}=\cup_{p>1} A_{p}^{+}$there exists $C$ such that

$$
\int_{\mathbb{R}}\left|M^{+} f(x)\right|^{p} w(x) d x \leq C \int_{\mathbb{R}}\left(f^{+, \#}(x)\right)^{p} w(x) d x
$$

whenever the left hand side is finite.

As in [1] (see the proof of Theorem 5.1 (iv) in [1] ) we can easily obtain the following lemma.

Lemma 4.3. Let $-1<\alpha \leq 0$. Then, for all $s>\frac{1}{1+\alpha}$, there exists a constant $C$ independent of $N$ such that

$$
\left[\left|T_{N}^{\alpha} f\right|\right]^{+, \#}(x) \leq C M_{s}^{+} f(x) .
$$

The restriction $s>1 /(1+\alpha)$ appears since the kernels $K_{N}^{\alpha}$ satisfy $D_{r}$ for $r<-1 / \alpha(1 /(1+\alpha)$ is the conjugate exponent of $-1 / \alpha)$. Now, we shall prove that the operators $T_{N}^{\alpha}$ are uniformly bounded in the weighted $L^{p}$ spaces.

Theorem 4.4. Let $-1<\alpha \leq 0, \frac{1}{1+\alpha}<p<\infty$ and $w \in A_{p(1+\alpha)}^{+}$. There exists a constant $C$ depending on $\alpha, p, \rho$ and $\left\|v_{k}\right\|_{\infty}$ such that

$$
\int\left|T_{N}^{\alpha} f(x)\right|^{p} w(x) d x \leq C \int|f(x)|^{p} w(x) d x
$$

for all $f \in L^{p}(w)$.

Proof: We know that $A_{p(1+\alpha)}^{+} \subset A_{p}^{+} \subset A_{\infty}^{+}$and that for each $w \in$ $A_{p(1+\alpha)}^{+}$there exists $t$ bigger than one such that $p(1+\alpha) / t>1$ and $w \in A_{p(1+\alpha) / t}^{+}([\mathbf{1 2}],[\mathbf{8}])$. On the other hand, since

$$
\left|T_{N}^{\alpha} f(x)\right| \leq 2\left\|v_{k}\right\|_{\infty}\left(N_{2}-N_{1}+1\right) \mathcal{M}_{\alpha}^{+} f(x),
$$

we get that for all $f \in L^{p}(w)$

$$
\begin{aligned}
\int_{\mathbb{R}}\left[M^{+}\left(\left|T_{N}^{\alpha} f\right|\right)(x)\right]^{p} w(x) d x & \leq C_{N} \int\left[M^{+}\left(\mathcal{M}_{\alpha}^{+} f\right)\right]^{p}(x) w(x) d x \\
& \leq C_{N} \int\left[\mathcal{M}_{\alpha}^{+} f\right]^{p}(x) w(x) d x \\
& \leq C_{N} \int|f|^{p}(x) w(x) d x<\infty
\end{aligned}
$$


Thus, applying Theorem 4.2 and Lemma 4.3 with $s=t /(1+\alpha)$ we have

$$
\begin{aligned}
\int\left|T_{N}^{\alpha} f(x)\right|^{p} w(x) d x & \leq C \int\left[M^{+}\left(\left|T_{N}^{\alpha} f\right|\right)(x)\right]^{p} w(x) d x \\
& \leq C \int\left[\left(\left|T_{N}^{\alpha} f\right|\right)^{+, \#}(x)\right]^{p} w(x) d x \\
& \leq C \int\left[M_{s}^{+} f(x)\right]^{p} w(x) d x \\
& =C \int\left[M^{+}\left(|f|^{s}\right)(x)\right]^{p / s} w(x) d x \\
& \leq C \int|f(x)|^{p} w(x) d x,
\end{aligned}
$$

where in the last inequality we have applied that $M^{+}$is bounded in $L^{p / s}(w)$ since $w \in A_{p / s}^{+}$.

\section{Proof of Theorem 1.6}

We shall prove the $L^{p}$ boundedness of the operator $T^{*}$. For $N=$ $\left(N_{1}, N_{2}\right)$, we shall use $T_{N_{1}, N_{2}}^{\alpha}$ to denote the operator $T_{N}^{\alpha}$. We start proving a pointwise estimate for the operators

$$
T_{M}^{*} f(x)=\sup _{\left|N_{1}\right|,\left|N_{2}\right| \leq M}\left|T_{N_{1}, N_{2}}^{\alpha} f(x)\right| .
$$

Theorem 5.1. Let $-1<\alpha \leq 0$. For each $s>1 /(1+\alpha)$ there exists a constant $C$ depending on $\alpha, \bar{s}, \rho$ and $\left\|v_{k}\right\|_{\infty}$ such that for every $x \in \mathbb{R}$ and every $M>0$

$$
T_{M}^{*} f(x) \leq C\left[M^{+}\left(\left|T_{-M, M}^{\alpha} f\right|\right)(x)+M_{s}^{+} f(x)+\mathcal{M}_{\alpha}^{+} f(x)\right] .
$$

Proof: Since the operators $T_{N_{1}, N_{2}}^{\alpha}$ are given by convolution, they are invariant under translations, and therefore it is enough to prove the theorem for $x=0$. Notice that

$$
T_{N_{1}, N_{2}}^{\alpha} f(x)=T_{N_{1}, M}^{\alpha} f(x)-T_{N_{2}+1, M}^{\alpha} f(x) .
$$

Then, it will suffice to estimate $\left|T_{m, M}^{\alpha} f(0)\right|$ for $|m| \leq M$ with constants independent of $m$ and $M$. Let us split $f$ as $f=f_{1}+f_{2}+f_{3}$, where $f_{1}=f \chi_{\left(0, \varepsilon_{m-1}\right)}, f_{2}=f \chi_{\left(\varepsilon_{m-1}, \infty\right)}$ and $f_{3}=f \chi_{(-\infty, 0)}$. First, notice that 
$T_{m, M}^{\alpha}\left(f_{3}\right)(0)=0$. Then

$$
\begin{aligned}
\left|T_{m, M}^{\alpha} f(0)\right| & \leq\left|T_{m, M}^{\alpha} f_{1}(0)\right|+\left|T_{m, M}^{\alpha} f_{2}(0)\right| \\
& =I+I I .
\end{aligned}
$$

It is clear that

$$
\begin{aligned}
I & \leq C \sum_{k=m-1}^{M} \frac{1}{\varepsilon_{k}^{1+\alpha}} \int_{0}^{\varepsilon_{m-1}}\left(\varepsilon_{k}-y\right)^{\alpha}|f(y)| d y \\
& \leq C \sum_{k=m-1}^{M}\left(\frac{1}{\rho}\right)^{(1+\alpha)(k-m+1)}\left(\frac{1}{\varepsilon_{m-1}^{1+\alpha}} \int_{0}^{\varepsilon_{m-1}}|f(y)|\left(\varepsilon_{m-1}-y\right)^{\alpha} d y\right) \\
& \leq C \mathcal{M}_{\alpha}^{+} f(0) .
\end{aligned}
$$

On the other hand

$$
\begin{aligned}
I I= & \frac{1}{\varepsilon_{m-1-\beta}} \int_{0}^{\varepsilon_{m-1-\beta}}\left|T_{m, M}^{\alpha} f_{2}(0)\right| d x \\
\leq & \frac{1}{\varepsilon_{m-1-\beta}} \int_{0}^{\varepsilon_{m-1-\beta}}\left|T_{-M, M}^{\alpha} f(x)\right| d x \\
& +\frac{1}{\varepsilon_{m-1-\beta}} \int_{0}^{\varepsilon_{m-1-\beta}}\left|T_{-M, M}^{\alpha} f_{1}(x)\right| d x \\
& +\frac{1}{\varepsilon_{m-1-\beta}} \int_{0}^{\varepsilon_{m-1-\beta}}\left|T_{m, M}^{\alpha} f_{2}(0)-T_{m, M}^{\alpha} f_{2}(x)\right| d x \\
& +\frac{1}{\varepsilon_{m-1-\beta}} \int_{0}^{\varepsilon_{m-1-\beta}}\left|T_{-M, m-1}^{\alpha} f_{2}(x)\right| d x \\
= & A_{1}+A_{2}+A_{3}+A_{4} .
\end{aligned}
$$

(If $m=-M$ we understand that $A_{4}=0$.) It is obvious that

$$
A_{1} \leq M^{+}\left(\left|T_{-M, M}^{\alpha} f\right|\right)(0) .
$$


For the second term, we use the uniform boundedness on $L^{s}$ of the operators $T_{-M, M}$ given in Theorem 4.1 (ii). Thus

$$
\begin{aligned}
A_{2} & \leq\left(\frac{1}{\varepsilon_{m-1-\beta}} \int_{0}^{\varepsilon_{m-1-\beta}}\left|T_{-M, M}^{\alpha} f_{1}(x)\right|^{s} d x\right)^{1 / s} \\
& \leq C\left(\frac{1}{\varepsilon_{m-1-\beta}} \int_{0}^{\varepsilon_{m-1}}|f(x)|^{s} d x\right)^{1 / s} \leq C M_{s}^{+} f(0),
\end{aligned}
$$

where in the last inequality we have used condition (2.3). Now we estimate $A_{3}$. Let $x \in\left(0, \varepsilon_{m-1-\beta}\right), s>1 /(1+\alpha)$ and $1 / s+1 / s^{\prime}=1$, by Hölder inequality and Lemma 3.6 we have that

$$
\begin{aligned}
& \left|T_{m, M}^{\alpha} f_{2}(0)-T_{m, M}^{\alpha} f_{2}(x)\right| \\
& \quad=\left|\int_{y>\varepsilon_{m-1}}\left[K_{N}^{\alpha}(-y)-K_{N}^{\alpha}(x-y)\right] f_{2}(y) d y\right| \\
& \quad=\sum_{j=m-1}^{\infty}\left(\int_{\varepsilon_{j}}^{\varepsilon_{j+1}}\left|K_{N}^{\alpha}(x-y)-K_{N}^{\alpha}(-y)\right|^{s^{\prime}} d y\right)^{1 / s^{\prime}}\left(\int_{0}^{\varepsilon_{j+1}}|f(y)|^{s} d y\right)^{1 / s} \\
& \quad \leq C M_{s}^{+} f(0) .
\end{aligned}
$$

Finally, we estimate $A_{4}$. First, it is clear that

$A_{4} \leq \frac{C}{\varepsilon_{m-1-\beta}} \int_{0}^{\varepsilon_{m-1-\beta}} \sum_{k=-M}^{m-1} \frac{1}{\varepsilon_{k}^{1+\alpha}} \int_{\varepsilon_{m-1}}^{\infty}\left(\varepsilon_{k}+x-y\right)^{\alpha} \chi_{\left(x, x+\varepsilon_{k}\right)}(y)|f(y)| d y d x$.

By using (2.4) we get that for $x \in\left(0, \varepsilon_{m-1-\beta}\right)$ and $k \leq m-2, x+\varepsilon_{k} \leq$ $\varepsilon_{m-1-\beta}+\varepsilon_{m-2} \leq \varepsilon_{m-1}$. Therefore, the sum in the above inequality reduces to the term $k=m-1$. Thus, we have

$$
\begin{aligned}
A_{4} & \leq \frac{C}{\varepsilon_{m-1-\beta}} \int_{0}^{\varepsilon_{m-1-\beta}} \frac{1}{\varepsilon_{m-1}^{1+\alpha}} \int_{\varepsilon_{m-1}}^{x+\varepsilon_{m-1}}\left(\varepsilon_{m-1}+x-y\right)^{\alpha}|f(y)| d y d x \\
& \leq \frac{C}{\varepsilon_{m-1-\beta}} \int_{0}^{\varepsilon_{m-1-\beta}} \frac{1}{\left(x+\varepsilon_{m-1}\right)^{1+\alpha}} \int_{0}^{x+\varepsilon_{m-1}}\left(\varepsilon_{m-1}+x-y\right)^{\alpha}|f(y)| d y d x \\
& \leq C \mathcal{M}_{\alpha}^{+} f(0) .
\end{aligned}
$$

Putting all these inequalities together we are done. 
Proof of Theorem 1.6: As in Theorem 4.4, since $w \in A_{p(1+\alpha)}^{+}$there is an $s>1 /(1+\alpha)$ such that $p / s>1$ and $w \in A_{p / s}^{+}$. By Theorem 5.1 we have

$$
T_{M}^{*} f(x) \leq C\left[M^{+}\left(\left|T_{-M, M}^{\alpha} f\right|\right)(x)+M_{s}^{+} f(x)+\mathcal{M}_{\alpha}^{+} f(x)\right] .
$$

The operator $M_{s}^{+}$is bounded in $L^{p}(w)$ because $w \in A_{p / s}^{+}$. Theorems 1.4 and 4.4 and the relation $A_{p(1+\alpha)}^{+} \subset A_{p}^{+}$give the uniform boundedness of the others two operators. Consequently, letting $M$ increase to infinity, we see that the same holds for the operator $T^{*}$ and we are done.

\section{Proof of Theorem 1.7}

We begin studying the behavior of $T^{*}$ on the functions of compact support and average zero. For this, we shall need the following remark.

Remark 6.1. It is clear that $w \in A_{1}^{+}$implies the following condition: there exists $C$ such that for any $M>1$ and every interval $I=(a, a+h)$

$$
\int_{a-M h}^{a} w \leq C M h \operatorname{essinf}\{w(x): x \in I\} .
$$

It follows from this property that if $w$ satisfies $A_{1}^{+}$then the following onesided doubling property holds: there exists $C$ such that for any $M>1$

$$
\int_{a-M h}^{a+h} w \leq(C M+1) \int_{a}^{a+h} w .
$$

Lemma 6.2. Let a be supported on $I=\left(0, \varepsilon_{i}\right)$ and such that $\int_{I} a=0$. Let $-1<\alpha \leq 0$. Assume that $w$ is a weight such that $w^{r} \in A_{1}^{+}$for some $r>1 /(1+\alpha)$. Then there exists $C>0$, independent of $a$, such that

$$
\int_{z<-\varepsilon_{i+\beta}} T^{*} a(z) w(z) d z \leq C \int_{I}|a(z)| w(z) d z .
$$

Proof: Let us write

$$
\int_{z<-\varepsilon_{i+\beta}} T^{*} a(z) w(z) d z=\sum_{m=i+\beta}^{\infty} \int_{-\varepsilon_{m+1}}^{-\varepsilon_{m}} T^{*} a(z) w(z) d z .
$$

Let $z \in\left(-\varepsilon_{m+1},-\varepsilon_{m}\right)$. Then, for fixed $N \in \mathbb{Z}^{2}$ we get that

$$
\left|T_{N}^{\alpha} a(z)\right| \leq C \sum_{k=-\infty}^{\infty}\left|\int_{I}\left[\varphi_{k}(z-u)-\varphi_{k-1}(z-u)\right] a(u) d u\right| .
$$


Observe that if $z \in\left(-\varepsilon_{m+1},-\varepsilon_{m}\right)$ and $u \in I$ we have that $z-u \in$ $\left(-\varepsilon_{m+2},-\varepsilon_{m}\right)$. Then $z-u \in\left(-\varepsilon_{k}, 0\right)$ for all $k \geq m+2$ and $z-u \notin$ $\left(-\varepsilon_{k}, 0\right)$ for all $k \leq m$. Therefore, for all $z \in\left(-\varepsilon_{m+1},-\varepsilon_{m}\right)$,

$$
\begin{aligned}
\left|T_{N}^{\alpha} a(z)\right| \leq & C\left|\int_{I} \frac{\left(\varepsilon_{m+1}+z-u\right)^{\alpha}}{\varepsilon_{m+1}^{1+\alpha}} \chi_{\left(-\varepsilon_{m+1}, 0\right)}(z-u) a(u) d u\right| \\
& +C\left|\int_{I} \frac{\left(\varepsilon_{m+2}+z-u\right)^{\alpha}}{\varepsilon_{m+2}^{1+\alpha}} a(u) d u\right| \\
& +C \sum_{k=m+3}^{\infty}\left|\int_{I} \frac{\left(\varepsilon_{k}+z-u\right)^{\alpha}}{\varepsilon_{k}^{1+\alpha}} a(u) d u\right| \\
= & A_{m}(z)+B_{m}(z)+C_{m}(z) .
\end{aligned}
$$

Notice that

$$
\begin{aligned}
\int_{-\varepsilon_{m+1}}^{-\varepsilon_{m}} A_{m}(z) w(z) d z= & \int_{-\varepsilon_{m+1}}^{-\varepsilon_{m+1}+2 \varepsilon_{i}} \cdots+\int_{-\varepsilon_{m+1}+2 \varepsilon_{i}}^{-\varepsilon_{m}} \cdots \\
\leq & C \int_{-\varepsilon_{m+1}}^{-\varepsilon_{m+1}+2 \varepsilon_{i}} w(z) \int_{I} \frac{\left|\varepsilon_{m+1}+z-u\right|^{\alpha}}{\varepsilon_{m+1}^{1+\alpha}}|a(u)| d u d z \\
& +C \int_{-\varepsilon_{m+1}+2 \varepsilon_{i}}^{0} w(z)\left|\int_{I} \frac{\left(z+\varepsilon_{m+1}-u\right)^{\alpha}}{\varepsilon_{m+1}^{1+\alpha}} a(u) d u\right| d z
\end{aligned}
$$

Now, doing the same with $B_{m}(z)$ we get

$$
\begin{aligned}
\int_{-\varepsilon_{m+1}}^{-\varepsilon_{m}} B_{m}(z) w(z) d z \leq & \int_{-\varepsilon_{m+2}}^{-\varepsilon_{m+2}+2 \varepsilon_{i}} \cdots+\int_{-\varepsilon_{m+2}+2 \varepsilon_{i}}^{-\varepsilon_{m}} \cdots \\
\leq & C \int_{-\varepsilon_{m+2}}^{-\varepsilon_{m+2}+2 \varepsilon_{i}} w(z) \int_{I} \frac{\left|\varepsilon_{m+2}+z-u\right|^{\alpha}}{\varepsilon_{m+2}^{1+\alpha}}|a(u)| d u d z \\
& +C \int_{-\varepsilon_{m+2}+2 \varepsilon_{i}}^{0} w(z)\left|\int_{I} \frac{\left(z+\varepsilon_{m+2}-u\right)^{\alpha}}{\varepsilon_{m+2}^{1+\alpha}} a(u) d u\right| d z
\end{aligned}
$$


Consequently,

$$
\begin{aligned}
\int_{z<-\varepsilon_{i+\beta}} T^{*} a(z) w(z) d z & \sum_{m=i+\beta}^{\infty} \int_{-\varepsilon_{m+1}}^{\varepsilon_{m}}\left(A_{m}(z)+B_{m}(z)+C_{m}(z)\right) w(z) d z \\
\leq & C \sum_{m=i+\beta}^{\infty} \int_{-\varepsilon_{m+1}}^{-\varepsilon_{m+1}+2 \varepsilon_{i}} w(z) \int_{0}^{\varepsilon_{i}} \frac{\left|\varepsilon_{m+1}+z-u\right|^{\alpha}}{\varepsilon_{m+1}^{1+\alpha}}|a(u)| d u d z \\
& +C \sum_{m=i+\beta}^{\infty} \int_{-\varepsilon_{m+1}+2 \varepsilon_{i}}^{0} w(z)\left|\int_{0}^{\varepsilon_{i}} \frac{\left(z+\varepsilon_{m+1}-u\right)^{\alpha}}{\varepsilon_{m+1}^{1+\alpha}} a(u) d u\right| d z \\
& +C \sum_{m=i+\beta}^{\infty} \int_{-\varepsilon_{m+1}}^{-\varepsilon_{m}} w(z) \sum_{k=m+3}^{\infty}\left|\int_{0}^{\varepsilon_{i}} \frac{\left(\varepsilon_{k}+z-u\right)^{\alpha}}{\varepsilon_{k}^{1+\alpha}} a(u) d u\right| d z \\
= & I+I I+I I I .
\end{aligned}
$$

Now we shall prove that each sum is dominated by $C \int_{0}^{\varepsilon_{i}}|a(u)| w(u) d u$. By Fubini's theorem and Hölder inequality with exponents $r>1 /(1+$ $\alpha$ ) and $r^{\prime}, 1 / r+1 / r^{\prime}=1$, we obtain for the first sum the following inequalities:

$$
\begin{aligned}
I & \leq C \sum_{m=i+\beta}^{\infty} \int_{0}^{\varepsilon_{i}}|a(u)| \int_{-\varepsilon_{m+1}}^{-\varepsilon_{m+1}+2 \varepsilon_{i}} w(z) \frac{\left|\varepsilon_{m+1}+z-u\right|^{\alpha}}{\varepsilon_{m+1}^{1+\alpha}} d z d u \\
& \leq C \sum_{m=i+\beta}^{\infty} \int_{0}^{\varepsilon_{i}}|a(u)| \frac{1}{\varepsilon_{m+1}^{1+\alpha}}\left(\int_{-\varepsilon_{m+1}}^{-\varepsilon_{m+1}+2 \varepsilon_{i}} w^{r}(z) d z\right)^{1 / r}\left(2 \varepsilon_{i}-u\right)^{\alpha+1 / r^{\prime}} d u \\
& \leq C \sum_{m=i+\beta}^{\infty} \int_{0}^{\varepsilon_{i}}|a(u)| \frac{1}{\varepsilon_{m+1}^{1+\alpha}}\left(\int_{-\varepsilon_{m+1}}^{0} w^{r}(z) d z\right)^{1 / r} \varepsilon_{i}^{\alpha+1 / r^{\prime}} d u .
\end{aligned}
$$

Let us fix $r>1 /(1+\alpha)$ such that $w^{r} \in A_{1}^{+}$. By (6.1) we get that

$$
\begin{aligned}
I & \leq C \int_{0}^{\varepsilon_{i}}|a(u)| w(u) d u \sum_{m=i+\beta}^{\infty}\left(\frac{\varepsilon_{i}}{\varepsilon_{m+1}}\right)^{\alpha+1 / r^{\prime}} \\
& \leq C \int_{0}^{\varepsilon_{i}}|a(u)| w(u) d u .
\end{aligned}
$$


We notice that we have used twice that $\alpha r^{\prime}+1>0$. We shall estimate now the second sum. First we write $I I$ as

$C \sum_{m=i+\beta \ell=1}^{\infty} \sum_{-\varepsilon_{m+1}+2^{\ell} \varepsilon_{i}}^{\infty} \int_{0}^{-\varepsilon_{m+1}+2^{\ell+1} \varepsilon_{i}}\left|\int_{0}^{\varepsilon_{i}} a(u) \frac{\left(z+\varepsilon_{m+1}-u\right)^{\alpha}}{\varepsilon_{m+1}^{1+\alpha}} d u\right| w(z) \chi_{(-\infty, 0)}(z) d z$.

Using that $\int_{0}^{\varepsilon_{i}} a=0$, the mean value theorem and the fact that $z \in$ $\left(-\varepsilon_{m+1}+2^{\ell} \varepsilon_{i},-\varepsilon_{m+1}+2^{\ell+1} \varepsilon_{i}\right)$ we obtain that

$$
\begin{aligned}
\left|\int_{0}^{\varepsilon_{i}} a(u)\left(z+\varepsilon_{m+1}-u\right)^{\alpha} d u\right| & \leq C \int_{0}^{\varepsilon_{i}}|a(u)|\left(z+\varepsilon_{m+1}-u\right)^{\alpha-1} u d u \\
& \leq C \int_{0}^{\varepsilon_{i}}|a(u)|\left(2^{\ell} \varepsilon_{i}\right)^{\alpha-1} \varepsilon_{i} d u .
\end{aligned}
$$

Therefore, $I I$ is bounded by

$C \sum_{m=i+\beta}^{\infty} \frac{\left(\varepsilon_{i}\right)^{\alpha}}{\left(\varepsilon_{m+1}\right)^{1+\alpha}} \int_{0}^{\varepsilon_{i}}|a(u)| d u \sum_{\ell=1}^{\infty} 2^{\ell(\alpha-1)} \int_{-\varepsilon_{m+1}+2^{\ell} \varepsilon_{i}}^{-\varepsilon_{m+1}+2^{\ell+1} \varepsilon_{i}} w(z) \chi_{(-\infty, 0)}(z) d z$.

Let us fix again $r>1 /(1+\alpha)$ such that $w^{r} \in A_{1}^{+}$. By Hölder inequality and (6.1) we get

$$
\begin{aligned}
\int_{-\varepsilon_{m+1}+2^{\ell} \varepsilon_{i}}^{-\varepsilon_{m+1}+2^{\ell+1} \varepsilon_{i}} w(z) \chi_{(-\infty, 0)}(z) d z & \leq\left(\int_{-\varepsilon_{m+1}}^{0} w^{r}\right)^{1 / r}\left(2^{\ell} \varepsilon_{i}\right)^{1 / r^{\prime}} \\
& \leq C \varepsilon_{m+1}^{1 / r} \operatorname{essinf}_{x \in\left(0, \varepsilon_{i}\right)} w(x)\left(2^{\ell} \varepsilon_{i}\right)^{1 / r^{\prime}}
\end{aligned}
$$

and consequently

$$
\begin{aligned}
I I & \leq C \sum_{m=i+\beta}^{\infty}\left(\frac{\varepsilon_{i}}{\varepsilon_{m+1}}\right)^{\alpha+1 / r^{\prime}}\left(\int_{0}^{\varepsilon_{i}}|a(u)| w(u) d u\right) \sum_{\ell=1}^{\infty} 2^{\ell\left(\alpha-1+1 / r^{\prime}\right)} \\
& \leq C \int_{0}^{\varepsilon_{i}}|a(u)| w(u) d u .
\end{aligned}
$$

In order to estimate the third sum, we use again that $\int_{0}^{\varepsilon_{i}} a=0$ and the mean value theorem. Then we have

$$
\begin{aligned}
I I I & \leq C \sum_{m=i+\beta}^{\infty} \int_{-\varepsilon_{m+1}}^{-\varepsilon_{m}} w(z) \sum_{k=m+3}^{\infty} \int_{0}^{\varepsilon_{i}} \frac{\left(\varepsilon_{k}+z-u\right)^{\alpha-1}}{\varepsilon_{k}^{1+\alpha}} u|a(u)| d u d z \\
& \leq C \sum_{m=i+\beta}^{\infty} \sum_{k=m+3}^{\infty} \int_{0}^{\varepsilon_{i}} \varepsilon_{i}|a(u)| \int_{-\varepsilon_{m+1}}^{-\varepsilon_{m}} \frac{\left(\varepsilon_{k}+z-u\right)^{\alpha-1}}{\varepsilon_{k}^{1+\alpha}} w(z) d z d u .
\end{aligned}
$$


Obvious inequalities, (2.3), (2.4) and $w \in A_{1}^{+}$give for almost every $u \in$ $\left(0, \varepsilon_{i}\right)$

$$
\begin{aligned}
\int_{-\varepsilon_{m+1}}^{-\varepsilon_{m}}\left(\varepsilon_{k}+z-u\right)^{\alpha-1} w(z) d z & \leq\left(\varepsilon_{k}-\varepsilon_{m+1}-\varepsilon_{i}\right)^{\alpha-1} \int_{-\varepsilon_{m+1}}^{-\varepsilon_{m}} w(z) d z \\
& \leq C\left(\varepsilon_{m+1}+\varepsilon_{i}\right)\left(\varepsilon_{m+3}-\varepsilon_{m+1}-\varepsilon_{i}\right)^{\alpha-1} w(u) \\
& \leq C \varepsilon_{m+3}^{\alpha} w(u) .
\end{aligned}
$$

Consequently,

$$
\begin{aligned}
I I I & \leq C\left(\int_{0}^{\varepsilon_{i}}|a(u)| w(u) d u\right) \varepsilon_{i} \sum_{m=i+\beta}^{\infty} \varepsilon_{m+3}^{\alpha} \sum_{k=m+3}^{\infty} \frac{1}{\varepsilon_{k}^{1+\alpha}} \\
& \leq C\left(\int_{0}^{\varepsilon_{i}}|a(u)| w(u) d u\right) \varepsilon_{i} \sum_{m=i+\beta}^{\infty} \frac{1}{\varepsilon_{m+3}} \\
& \leq C \int_{0}^{\varepsilon_{i}}|a(u)| w(u) d u .
\end{aligned}
$$

Corollary 6.3. Let $-1<\alpha \leq 0$ and let $w$ be a weight such that $w^{r} \in A_{1}^{+}$ for some $r>1 /(1+\alpha)$. Let a be supported on $I=\left(x^{*}, x^{*}+h\right)$ and such that $\int_{I} a=0$. If $A=\rho^{2(\beta+1)}$ there exists $C$ independent of $x^{*}, h$ and $a$, such that

$$
\int_{z<x^{*}-A h} T^{*} a(z) w(z) d z \leq C \int_{I}|a(z)| w(z) d z .
$$

Proof: First notice that it is sufficient to prove the corollary for $x^{*}=0$. Choose $i$ such that $\varepsilon_{i-1} \leq h<\varepsilon_{i}$. Then $a$ is supported on $\left(0, \varepsilon_{i}\right)$ and has integral zero. Furthermore, by $(2.3),-A h<-\varepsilon_{i+\beta}$ and by the lemma

$$
\int_{z<-A h} T^{*} a(z) w(z) d z \leq \int_{z<-\varepsilon_{i+\beta}} T^{*} a(z) w(z) d z \leq C \int_{I}|a(z)| w(z) d z .
$$

Once we have Corollary 6.3 and Theorem 1.6 the proof of Theorem 1.7 is straightforward (see for instance $[\mathbf{1}]$ ). We include it for the sake of completeness.

Proof of Theorem 1.7: Let $O_{\lambda}=\left\{x: M^{+} f(x)>\lambda\right\}$. It is well known that if $\left\{I_{i}\right\}$ are the connected components of $O_{\lambda}$, then $\lambda=\frac{1}{\left|I_{i}\right|} \int_{I_{i}} f=f_{I_{i}}$. We decompose $f$ as

$$
f=f \chi_{\mathbb{R} \backslash O_{\lambda}}+\sum f_{I_{i}} \chi_{I_{i}}+\sum\left(f-f_{I_{i}}\right) \chi_{I_{i}} .
$$


As usual $f \chi_{\mathbb{R} \backslash O_{\lambda}}+\sum f_{I_{i}} \chi_{I_{i}}$ will be denoted by $g$ and $\sum\left(f-f_{I_{i}}\right) \chi_{I_{i}}=$ $\sum b_{i}$ by $b$. Observe that each $b_{i}$ has support on $I_{i}$ and average zero. Now,

$$
\begin{aligned}
\int_{\mathbb{R}}|g(y)| w(y) d y & \leq \int_{\mathbb{R} \backslash O_{\lambda}}|f(y)| w(y) d y+\sum w\left(I_{i}\right) f_{I_{i}} \\
& =\int_{\mathbb{R} \backslash O_{\lambda}}|f(y)| w(y) d y+\lambda \sum w\left(I_{i}\right) \\
& =\int_{\mathbb{R} \backslash O_{\lambda}}|f(y)| w(y) d y+\lambda w\left(O_{\lambda}\right) \\
& \leq C \int_{\mathbb{R}}|f(y)| w(y) d y,
\end{aligned}
$$

because the operator $M^{+} f$ is of weak type $(1,1)$ with respect to $w$.

For each interval $I=(a, a+h)$, let us denote by $I^{*}$ the interval $(a-A h, a+h)$, where $A=\rho^{2(\beta+1)}$. We also denote by $\widetilde{O_{\lambda}}$ the union of all the intervals $I_{i}^{*}$. Observe that

$$
\begin{aligned}
w\left(\left\{x: T^{*} f(x)>\lambda\right\}\right) \leq & w\left(\left\{x: T^{*} g(x)>\lambda / 2\right\}\right)+w\left(\widetilde{O_{\lambda}}\right) \\
& +w\left(\left\{x \notin \widetilde{O_{\lambda}}: T^{*} b(x)>\lambda / 2\right\}\right)=I+I I+I I I .
\end{aligned}
$$

The one-sided doubling property of the weight and the weak type $(1,1)$ inequality for $M^{+}$give

$$
I I=w\left(\cup_{i} I_{i}^{*}\right) \leq C w\left(O_{\lambda}\right) \leq \frac{C}{\lambda} \int|f(y)| w(y) d y .
$$

On the other hand, since $A_{1}^{+}$implies condition $A_{p}^{+}$for any $p>1 /(1+\alpha)$, Theorem 1.6 implies that $T^{*}$ is a bounded operator in $L^{p}(w)$. Then we have

$$
\begin{aligned}
w\left(\left\{x: T^{*} g(x)>\lambda / 2\right\}\right) & \leq \frac{C}{\lambda^{p}} \int\left(T^{*} g(y)\right)^{p} w(y) d y \leq \frac{C}{\lambda^{p}} \int|g(y)|^{p} w(y) d y \\
& \leq \frac{C}{\lambda} \int|g(y)| w(y) d y \leq \frac{C}{\lambda} \int|f(y)| w(y) d y .
\end{aligned}
$$


Observe that in the last two inequalities we have used $|g| \leq \lambda$ and (6.4). Finally, by using Corollary 6.3 and the one-sided nature of the operator $T^{*}$, we have

$$
\begin{aligned}
I I I & \leq \frac{C}{\lambda} \int_{\mathbb{R} \backslash \widetilde{O_{\lambda}}} T^{*} b(x) w(x) d x \leq \frac{C}{\lambda} \sum_{i} \int_{\mathbb{R} \backslash I_{i}^{*}} T^{*} b_{i}(x) w(x) d x \\
& \leq \frac{C}{\lambda} \sum_{i} \int_{I_{i}}\left|b_{i}(x)\right| w(x) d x .
\end{aligned}
$$

Since the $I_{i}$ 's are disjoint and $b(x)=b_{i}(x)$ on each $I_{i}$ the last term is bounded by

$$
\frac{C}{\lambda} \int|b(x)| w(x) d x=\frac{C}{\lambda} \int|f(x)-g(x)| w(x) d x \leq \frac{C}{\lambda} \int|f(x)| w(x) d x .
$$

\section{Proof of Theorem $\mathbf{1 . 9}$}

By Theorems 1.6 and 1.7 it is clear that it suffices to prove the a.e. convergence in the Schwartz's class $\mathcal{S}$.

Theorem 7.1. The functions $T_{N}^{\alpha} \psi(x)$ converge for all $\psi \in \mathcal{S}$ and for every $x \in \mathbb{R}$ as $N=\left(N_{1}, N_{2}\right)$ tends to $(-\infty,+\infty)$. Further, if $\psi$ has compact support and $K^{\alpha}(x)=\sum_{k=-\infty}^{+\infty} v_{k}\left(\varphi_{k}(x)-\varphi_{k-1}(x)\right)$ then

$$
\lim _{N \rightarrow(-\infty,+\infty)} T_{N}^{\alpha} \psi(x)=K^{\alpha} * \psi(x)
$$

for all $x$ outside the support of $\psi \in \mathcal{S}$.

Proof: It will suffice to show that $T_{-M, 0}^{\alpha} \psi(x)$ and $T_{0, M}^{\alpha} \psi(x)$ converge as $M \rightarrow+\infty$. In fact, we shall prove that

$$
\begin{aligned}
& \left|T_{-M, 0}^{\alpha} \psi(x)-T_{-N, 0}^{\alpha} \psi(x)\right|+\left|T_{0, M}^{\alpha} \psi(x)-T_{0, N}^{\alpha} \psi(x)\right| \\
& \quad=\left|T_{-M,-N-1}^{\alpha} \psi(x)\right|+\left|T_{N+1, M}^{\alpha} \psi(x)\right|=I+I I
\end{aligned}
$$

is small for $N<M$ and $N$ big enough. First, let us observe that from (2.3) we have that for all $s>0$ there exists a positive constant $C$ such that for each $m \leq n$

$$
\sum_{k=-\infty}^{n} \varepsilon_{k}^{s} \leq C \varepsilon_{n}^{s} \quad \text { and } \quad \sum_{k=m}^{\infty} \frac{1}{\varepsilon_{k}^{s}} \leq C \frac{1}{\varepsilon_{m}^{s}} .
$$


Since $\int K_{N}^{\alpha}=0$ for every $N \in \mathbb{Z}^{2}$, by using the mean value theorem and (7.2) we get that

$$
\begin{aligned}
I & =\left|\int K_{-M,-N-1}^{\alpha}(x-y)[\psi(y)-\psi(x)] d y\right| \\
& \leq 2\left\|\psi^{\prime}\right\|_{L^{\infty}}\left\|v_{k}\right\|_{\infty} \int \sum_{k=-M-1}^{-N-1} \varphi_{k}(u)|u| d u \\
& \leq C \sum_{k=-M-1}^{-N-1} \frac{1}{\varepsilon_{k}^{\alpha}} \int_{-\varepsilon_{k}}^{0}\left(\varepsilon_{k}+u\right)^{\alpha} d u \leq C \sum_{k=-M-1}^{-N-1} \varepsilon_{k} \leq C \varepsilon_{-N-1},
\end{aligned}
$$

which is small when $N$ is big enough. In order to estimate $I I$, we choose a number $s>1 /(1+\alpha)$. By Hölder's inequality and using (7.2) again we have

$$
\begin{aligned}
I I & \leq 2\left\|v_{k}\right\|_{\infty} \int \sum_{k=N}^{M} \varphi_{k}(x-y)|\psi(y)| d y \\
& \leq C \sum_{k=N}^{M} \frac{1}{\varepsilon_{k}^{1+\alpha}} \int_{x}^{x+\varepsilon_{k}}|\psi(y)|\left(\varepsilon_{k}+x-y\right)^{\alpha} d y \\
& \leq C\|\psi\|_{L^{s}} \sum_{k=N}^{M} \frac{1}{\varepsilon_{k}^{1+\alpha}}\left(\int_{x}^{x+\varepsilon_{k}}\left(x+\varepsilon_{k}-y\right)^{\alpha s^{\prime}} d y\right)^{1 / s^{\prime}} \\
& \leq C\|\psi\|_{L^{s}} \sum_{k=N}^{M} \frac{1}{\varepsilon_{k}^{1 / s}} \leq \frac{C}{\varepsilon_{N}^{1 / s}}\|\psi\|_{L^{s}},
\end{aligned}
$$

which can be done small taking $N$ big enough.

Let us denote the support of $\psi$ by $\operatorname{supp}(\psi)$. Let $x \notin \operatorname{supp}(\psi)$. Then there exists $\delta>0$ such that $|x-y| \geq \delta$ for all $y \in \operatorname{supp}(\psi)$. Let $k_{0} \in \mathbb{Z}$ such that $\varepsilon_{k_{0}}<\delta \leq \varepsilon_{k_{0}+1}$. Therefore, from Lemma 3.3 and taking into account the support of $K_{N}^{\alpha}$ we get that

$$
\left|K_{N}^{\alpha}(x-y)\right| \leq \frac{C}{\delta}+C \sum_{k \geq k_{0}} \frac{\left(\varepsilon_{k+1}+x-y\right)^{\alpha}}{\varepsilon_{k+1}^{1+\alpha}} \chi_{\left(-\varepsilon_{k+1},-\varepsilon_{k}\right)}(x-y) .
$$


Observe that $g(y)=\frac{C}{\delta} \psi(y)$ is an integrable function. We shall see now that the function

$$
h(y)=\sum_{k \geq k_{0}} \frac{\left(\varepsilon_{k+1}+x-y\right)^{\alpha}}{\varepsilon_{k+1}^{1+\alpha}} \chi_{\left(-\varepsilon_{k+1},-\varepsilon_{k}\right)}(x-y) \psi(y)
$$

is also integrable. Let $s>\frac{1}{1+\alpha}$ and $s^{\prime}$ its conjugate exponent. By the Hölder inequality

$$
\begin{aligned}
\int|h(y)| d y & \leq\|\psi\|_{L^{s}} \sum_{k \geq k_{0}}\left(\int_{x+\varepsilon_{k}}^{x+\varepsilon_{k+1}} \frac{\left(\varepsilon_{k+1}+x-y\right)^{s^{\prime} \alpha}}{\varepsilon_{k+1}^{s^{\prime}(1+\alpha)}} d y\right)^{1 / s^{\prime}} \\
& \leq C\|\psi\|_{L^{s}} \sum_{k \geq k_{0}} \varepsilon_{k+1}^{-1 / s} \leq C\|\psi\|_{L^{s} \varepsilon_{k_{0}}^{-1 / s}}
\end{aligned}
$$

Now the integrability of $g$ and $h$ together with the dominated convergence theorem imply

$$
\lim _{N \rightarrow(-\infty,+\infty)} T_{N}^{\alpha} \psi(x)=K^{\alpha} * \psi(x)
$$

for all $x$ outside the support of $\psi \in \mathcal{S}$.

\section{References}

[1] A. L. Bernardis, M. Lorente, F. J. Martín-Reyes, M. T. Martínez, A. de la Torre, and J. L. Torrea, Differential transforms in weighted spaces, J. Fourier Anal. Appl. 12(1) (2006), 83-103.

[2] A. L. Bernardis and F. J. Martín-Reyes, Two weighted inequalities for convolution maximal operators, Publ. Mat. 46(1) (2002), 119-138.

[3] M. Broise, Y. Déniel, and Y. Derriennic, Réarrangement, inégalités maximales et théorèmes ergodiques fractionnaires, Ann. Inst. Fourier (Grenoble) 39(3) (1989), 689-714.

[4] J. García-Cuerva and J. L. Rubio de Francia, "Weighted norm inequalities and related topics", North-Holland Mathematics Studies 116, Notas de Matemática 104, North-Holland Publishing Co., Amsterdam, 1985.

[5] R. Irmisch, Punktweise Ergodensätze für $(C, \alpha)$-Verfahren, $0<$ $\alpha<1$, Dissertation, Fachbereich Mathematik, TH Darmstadt (1980).

[6] R. L. Jones and J. Rosenblatt, Differential and ergodic transforms, Math. Ann. 323(3) (2002), 525-546. 
[7] W. B. Jurkat and J. L. Troutman, Maximal inequalities related to generalized a.e. continuity, Trans. Amer. Math. Soc. 252 (1979), 49-64.

[8] F. J. Martín-Reyes, New proofs of weighted inequalities for the one-sided Hardy-Littlewood maximal functions, Proc. Amer. Math. Soc. 117(3) (1993), 691-698.

[9] F. J. Martín-Reyes, P. Ortega Salvador, and A. De la ToRRE, Weighted inequalities for one-sided maximal functions, Trans. Amer. Math. Soc. 319(2) (1990), 517-534.

[10] F. J. Martín-Reyes And A. DE LA Torre, One-sided BMO spaces, J. London Math. Soc. (2) 49(3) (1994), 529-542.

[11] F. J. Martín-Reyes And A. De LA Torre, Some weighted inequalities for general one-sided maximal operators, Studia Math. 122(1) (1997), 1-14.

[12] E. SAWYER, Weighted inequalities for the one-sided Hardy-Littlewood] maximal functions, Trans. Amer. Math. Soc. 297(1) (1986), $53-61$.

[13] A. De la Torre And J. L. Torrea, One-sided discrete square function, Studia Math. 156(3) (2003), 243-260.

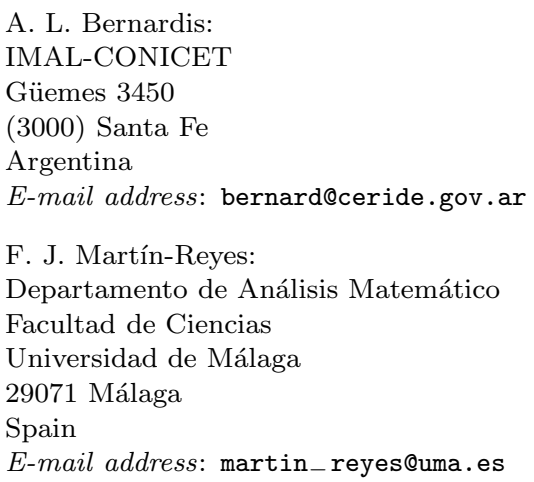

Primera versió rebuda el 5 d'octubre de 2006, darrera versió rebuda el 10 d'octubre de 2007. 